\title{
Medicina basada en evidencia (MBE). Contras
}

\author{
A. Berguer' ${ }^{1}$ R. Berguer ${ }^{2}$
}

\begin{abstract}
Resumen: El método de la llamada Medicina Basada en la Evidencia (MBE), se ha convertido en los últimos diez años en el paradigma de investigación clínica en las Ciencias de la Salud. Basada en métodos epidemiológicos y estadísticos, con antecedentes en la agronomía y emparentada filosóficamente con el método hipoteticodeductivo, la MBE sé esta estableciendo como la única doctrina que valide el trabajo científico de los profesionales, despreciando los paradigmas científicos que dieron como resultado los avances sensacionales que la medicina ha experimentado en nuestra generación.

Entendemos que la MBE debe suponer una ayuda para a que el fruto de la experiencia se concrete en lo posible de manera sistematica y objetiva en recomendaciones prácticas, no que una conclusión genérica, obtenida de un grupo de pacientes, se convierta en una recomendación de actuación específica sobre un enfermo en concreto.
\end{abstract}

Palabras clave: Medicina basada en la evidencia. Contras.

\begin{abstract}
The method of the "so called" Evidence Based Medicine (EBM), ha become in the last ten years in the paradigm of the clinical investigation in Health Sciences. Based on epidemiologic and statistic methods, with antecedents in agronomy and phylosophically linked with the hypothetic-deductive method, the EBM is being stablished as the unique doctrin that vallidates the scientigic work of these professionals, mis Reading the Scientifi paradigms that resulted the great advances that the Medicine han experienced in ou generation. We feel that the EBM must be a help por the experience to concrete in a systematic and objetive way in practical recommedations, not a gneric conclusion, obtained from a group of patients, will become a specific actuation recommedation of a patient.
\end{abstract}

Key words: Evidence Based Medicine; Disadvantages.

\section{Breve repaso de la Epistemología de la Ciencia}

Dos corrientes filosóficas antagónicas se disputan el rigor de verificar la verdad desde hace 23 siglos. Los

1 Jefe de Servicio de Cirugía Maxilofacial del Hospital Clínico San Carlos. Madrid.Prof.asoc. Depart. Cirugía de la Univ.Complutense. Madrid. España.

2 Jefe de Servicio de Cirugía Vascular del Centro Médico de Detroit y Prof. de Cirugía de la Univers. Wayne State de Detroit. Mi. EE.UU.

Correspondencia:

A.Berguer

Avda.Pablo Iglesias,15-entrepl. 28003 Madrid. España antecedentes de posturas científicas contrapuestas vienen, desde el siglo III a. C., con la Escuela Empírica de Alejandría fundada por Filino de Cos de un lado, y la Escuela Dogmática o Racional del otro. Estas filosofías contrapuestas, el Racionalismo Dogmático y el Empirismo Inductivo, se combinan en la práctica médica diaria a fin de construir las explicaciones sencillas de causa y efecto que constituyen nuestra interpretación del problema que tiene un enfermo.

El pensamiento «mágico», con sus signos premonitorios y la práctica de la 〈adivinación» fue el sistema de búsqueda de la verdad y de anticipación de eventos utilizado por el hombre desde su aparición en la tierra hasta el siglo $\mathrm{V}$ a. $\mathrm{C}$. El resto del pensamiento científico surgido a lo largo de la historia de la humanidad, 
podemos englobarlo fundamentalmente en las dos grandes tendencias amiba mencionadas: el Racionalismo 0 Método Deductivo y el Empirismo o Método Inductivo. El primero de ellos nace con Platón y Aristóteles, teniendo su base fundamental en la geometría Euclidiana, con arraigo entre los escolásticos medievales, modemizado por Descartes y Leibniz y respaldado como método Axiomático-Deductivo por Kant. Su fundamento está en que, a partir de una serie de enunciados racionales establecidos como axiomas, se deducen por implicación lógica una serie de teoremas o leyes de carácter general. No hay duda que esta forma de pensamiento racional tuvo que evolucionar a lo largo de la historia por cuanto nuevos descubrimientos fueron demostrando que algunos axiomas no eran verdaderos y, por tanto, las leyes deducidas tampoco lo eran.

El método Hipotético-Deductivo o también llamado Positivismo Lógico, preconizado por el grupo del Círculo de Viena en 1923 (Wittgenstein, Carnap y Popper), hay que considerarlo como el último empuje en la evolución del método racionalista que, guardando similares principios, introduce, a través de su propia denominación, lo que podníamos llamar un factor de «umildad»en el antaño categórico axiomáticodeductivo, dada la relatividad de las verdades «a prioris, Sin embargo, es radical a la hora de considerar que su metodología es la única que presupone seriedad científica.

En cualquier caso, hemos de tener en cuenta que el Método Racionalista puede establecer conclusiones válidas que no necesariamente han de ser verdaderas permanentemente; asimismo, puede demostrar la falsedad de una hipótesis, no su verdad. Hay que considerar también que el racionalismo puro no es válido para poder descubrir hechos nuevos, ya que hay que inventar, intuir, una relación antes de poder demostrarla como verdadera o falsa. Popper, introdujo una garantía mayor del desarrollo de este método con el principio de «erificabilidad», es decir, el Falsacionismo, que tendría la capacidad de demostrar la falsedad de una teoría por la existencia de enunciados lógicamente incompatibles con ella.

El Empirismo o Método Inductivo nace en la Grecia del III a.C., es desarrollado como sistema por Bacon y utilizado en la enunciación de la mecánica Celeste por Copérnico, Galileo, Kepler y Newton. Los enunciados observacionales del empinismo, snihil in intellectu nisi prius in sensu» ("nada en el intelecto que no haya pasado antes por los sentidos"), contravinieron en su día el criterio al que se había llegado a través del método racionalista por parte de Aristóteles, del mismo modo que, posteriormente la teoría de la relatividad de Einstein, desbordo la teoría de la gravitación universal de Newton. Su reflejo en la Medicina fueron los experimentos y descubrimientos de Claude Bernard sobre la digestion de los alimentos. Curiosamente, un grupo de médicos coetáneos franceses encabezados por Pierre Alexander Louis, propugnaron hacia 1830 el método racionalista que ellos llamaban «Medecine d'Observation»en París, aplicando métodos numéricos que podríamos considerar como el embrión de la actual MBE. El propio Bernard cuestionó como válidos estos métodos que, como él dijo: son «como adivinar cuantas personas hay en una casa por la cantidad de humo que sale de la chimenea».

Nadie puede negar que la investigación empírica es responsable de los descubrimientos de la fisiopatología aceptada hoy en día, de la eficacia de muchos fármacos, de técnicas concretas que han resultado en los enormes beneficios de tratamientos médicos y quirúrgicos y, en general, en la mayor parte de los avances de la medicina moderna.

De maravilloso recuerdo es el descubrimiento de la penicilina por Fleming, observando la inhibición bacteriana por hongos en las placas de Petri que había dejado en el dintel de la ventana de su laboratorio. No menos podemos decir del efecto anticoagulante de la cumarina, deducido de la observación de los efectos del veneno de rata en un suicidio humano. El hallazgo de la capacidad quimioterápica anticancerígena del cisplatino, fue apreciada cuando se estudiaban los efectos de pasar una corriente eléctrica a través de un medio de cultivo bacteriano; la inhibición que se observó alrededor de los electrodos no se debía a la comiente que circulaba entre ellos, sino que un astuto observador intuyó correctamente que la inhibición se debía al platino que se desprendía de los electrodos y se diluía en el medio de cultivo. Las prótesis arteriales concebidas por Blakemore, nacieron de la observación de que una sutura de nylon que atravesaba la aurícula de un perro no se había cubierto de trombo, como era de esperar, sino de una membrana lisa y limpia como un endotelio.

Lane en 1914, observó radiológicamente la consolidación ósea directa de las fracturas, que no fue demostrada clínicamente por Key hasta 1932. Danis en 1949, sentó las bases que habrían de regir la fijación rígida en las fracturas óseas de los huesos largos para obtener una consolidación directa, aunque hubiera que esperar hasta 1963 para que Schenk y Willenegger, la demostraran histológicamente.

Estos ejemplos y muchos otros, muestran que en la ciencia médica el empirismo no se ha nutrido de descubrimientos casuales, sino de la suma y conjunción en esas personas o equipos investigadores, de una aguda observación y una mente preparada. Asimismo, no es menos cierta la utilidad hasta el momento, de muchos actos diagnósticos o terapéuticos habituales sin necesidad de demostraciones epidemiológicas o formulaciones bioestadísticas. Valgan ejemplos tan claros como la utilidad de cateterizar una vejiga con obstrucción urinaria aguda, el drenaje de un absceso, la reducción e inmovilización de una fractura, o la inserción de un marcapasos en un bloqueo sintomático cardíaco. 
Con el nacimiento de la epidemiología y de la bioestadística se enriqueció la investigación médica, pero al tiempo también comenzaron a polarizarse las tendencias en uno y otro sentido, que nos han llevado a la situación actual. Mientras unos consideran que con el método inductivo y la ayuda de las ciencias formales auxiliares y sus instrumentos se puede llevar a cabo una argumentación científica correcta en gran parte de la investigación médica, otros estiman que solamente los métodos epidemiológicos y estadísticos pueden aportar rigor y criterio de veracidad a las investigaciones. Nosotros estimamos que ambas son fases de un mismo proceso, no procesos independientes y que han de suponer una retroalimentación secuencial del conjunto de la investigación.

\section{Resumen histórico de la MBE}

Ya mencionamos que el Grupo "Medecine d'Observation" en París representa el antecedente histórico de la defensa del método deductivo en la investigación médica. Las herramientas fundamentales de la MBE, los ensayos aleatorios y el análisis estadístico frequentista (el valor $\mathrm{p}$ de Fisher) (Goodman), 1, 2 de hecho, fueron desarrolladas y utilizadas por las escuelas de agronomía experimental, en la segunda y tercera década del siglo pasado. Es conveniente recordar esta estirpe porque el sujeto de estos métodos eran plantas cuya uniformidad y organización interactiva se contraponen a la variabilidad individual de los organismos humanos con fisiopatología alterada. El comienzo formal de estos métodos de análisis en medicina humana fueron los tres ensayos clínicos que Sir Austen Bradford Hill publicó en 1952 y que demostraron la eficacia de la estreptomicina para el tratamiento de la tuberculosis. Dos universidades sajonas son consideradas como la cuna de la MBE, la Universidad de Oxford en Inglaterra y la McMasters en Canada con las publicaciones pioneras de Cochrane en 1972, ${ }^{3}$ y de Sakett en 1979. ${ }^{4}$ En la Universidad de Oxford, se formo un grupo de trabajo, que fue el embrión de lo que en 1992 se llamaría el Centro Cochrane, el «emplo»de la MBE, así llamado en honor de Archibald Cochrane a quien se considera el fundador de la epidemiología modema. Llama la atención cuando se analizan los juicios de valor que el propio Sackett, ${ }^{5}$ hace de la MBE y del método empírico mostrándose mucho más respetuoso en la consideración de la experiencia acumulada y de la inteligencia clínica individual de lo que se muestran muchos de los «evangelistas»de este nuevo método como Harrington, 6 Jadad, ${ }^{7}$ Labréze, ${ }^{8}$ y otros.

No es nuestra misión en este artículo describir minuciosamente los contenidos, método de trabajo y características propias de la MBE, lo que debe corresponder sobre todo a quienes han de apoyarla en el otro artículo contrapuesto esta «controversia». Simplemen- te vamos a enunciar sus principios clasificatorios cualitativos y de valoración. Debemos en todo ello considerar la pomposidad semántica que supone denominarse de ese modo (Medicina «Basada en Evidencia»), con visos de certezas que excluyen otros métodos de busca de la verdad; tanto es así que, algunos ofendidos por este acto de soberbia, sugieren que se utilicen las mismas siglas (MBE) para rebautizar este movimiento como Medicina Basada en Epidemiología y otros han sugerido que el nombre adecuado a la función que realiza sería el de «Medicina Basada en Guías de Práctica Clínica». Esta expresión en el momento actual también sería poco afortunada, porque hasta el momento son escasas las Guías de Práctica Clínica con base en la MBE.

\section{Herramientas de la MBE}

La MBE sienta las bases para la validez de los estudios en tres grupos, según se correspondan con la evaluación de Pruebas Diagnósticas, el Pronóstico de Enfermedades o la Eficacia de los Tratamientos, precisando para ello los diseños de estudio adecuados a cada objetivo. A su vez cada uno de estos estudios deberá reunir unas condiciones para que se le considere que garantizan el «igor científico», Con todas estas características establecen los llamados Niveles de «Evidencia»(A,B,C y D), correspondiendo al último la «no evidencia».

Las herramientas fundamentales que utiliza la MBE son los Ensayos Clínicos Randomizados (ECR); es decir, con asignación aleatoria a los distintos grupos de ensayo y el Meta-Análisis (MA), como expresión no de una revisión sistemática de la literatura, sino como análisis de estudios realizados con una metodología similar. Con estas dos herramientas estiman conseguir las «irtudes» de la eliminación de sesgos a través de los «ciegos», tanto de todo el ensayo o de sus partes, una mayor significación estadística derivada de grandes tamaños muestrales y una mayor «uniformidad»en las evaluaciones.

En su aplicación práctica y con respecto a los ECR, hemos de advertir que están sometidos a múltiples sesgos potenciales que pueden comprometer su validez $\mathrm{y}$, a pesar de la existencia de instrumentos de evaluación de la propia calidad del diseño de los mismos, no hay un «gold estándar» para determinar la validez real de un ensayo clínico, como también es cuestionable la calidad de la publicación del mismo, a pesar de los diversos cuestionarios publicados hasta el momento (Estrada y Oliva). ${ }^{9}$ En cuanto al Meta-Análisis, el hecho del empleo de amplios estudios puede mejorar, pero no necesariamente eliminar errores pequeños del azar, sobre todo cuando existen diferencias de aglutinar los resultados procedentes de distintos estudios de calidad metodológica dispar. Si los 
criterios de inclusión son amplios, tal y como fueron empleados en los primeros Meta-Análisis utilizados en psicología y psiquiatría, la impureza y falta de uniformidad de datos confunden, en lugar de definir la respuesta. Sobre ello estimamos interesantes las reflexiones del histórico trabajo de Eysenck. ${ }^{10}$

$\mathrm{Al}$ respecto de lo comentado queremos poner un ejemplo muy significativo puesto de manifiesto por Schlemper y cols, ${ }^{11}$ respecto a las diferencias de criterio a la hora de diagnosticar un carcinoma gástrico entre patólogos japoneses y occidentales. Los japoneses presentaban unos resultados de tratamiento mucho mejores porque diagnosticaban como neoplasias lo que para los patólogos occidentales eran displasias o atipias. Esta carencia de rigor y uniformidad en el protocolo, como primera fase de un estudio, vicia de por sí, cualquier resto de las fases del estudio.

En algunos estudios de MBE inoportunamente aúnan síntomas de enfermedades que tienen etiologías diferentes, con lo cual sus conclusiones pueden ser erróneas. Otros estudios incluyen una variable que define el estadio de una patología y omiten otras variables «de confusión»que tienen la misma o mayor relevancia en la evolución de una enfermedad. Valga el ejemplo de los grandes estudios randomizados de la enfermedad carotídea el del ACAS, ${ }^{12}$ y NASCET; ${ }^{13}$ Ambos clasificaron las lesiones carotídeas internas, según su porcentaje de estenosis. Cuando estos estudios fueron diseñados ya se sabía, por publicaciones científicas de rigurosa calidad, que los infartos cerebrales que aparecían en pacientes con placas ateromatosas carotídeas eran causados en la gran mayonía de casos por embolización (tromboémbolo) a partir de la placa y no por el bajo flujo. Se sabía también, que las placas antiguas son de mayor tamaño que las nuevas y que, además, muestran con mayor frecuencia que estas ultimas características degenerativas (ulceración, trombo en superficie, hemorragia intraplaca). Del mismo modo se conocía que las lesiones carotídeas secundarias a hiperplasia de la íntima de superficies lisas son menos mórbidas que los ateromas ulcerados del mismo tamaño y grado oclusivo, (Imparato y cols.), ${ }^{14}$ (Dixon y cols.), ${ }^{15}$ Geroulakos y cols. ${ }^{16}$ En resumen, para estratificar la enfermedad carotídea, estas características (ulceración, heterogeneidad de composición, etc.) deberían de haber sido incluidas entre las variables a estudiar. Por el contrario se construyó una clasificación incorrecta y excesivamente simplificada por porcentajes de estenosis y desdeñaron los factores referidos, que son tan importantes o más que el grado de estenosis, para determinar el futuro de un enfermo carotídeo.

Este desdén por esquemas fisiopatológicos conocidos de antemano pero no adquiridos por estudios randomizados, es parte del estilo de la aplicación errónea de los conceptos propios de la epidemiología; tra- tando un problema como si fuese una "caja negra electrónica". Se define la variable a estudiar, se randomiza una muestra adecuada de la población y del análisis estadístico de los datos se deduce la probabilidad de asociación o de eficacia.

En cuanto a los MA, queremos poner un ejemplo reciente en Cirugía Oral y Maxilofacial, como es el realizado comparativamente sobre la efectividad de las técnicas quirúrgicas de artrocentésis y artroscopia en los desórdenes de la articulación temporomandibular (ATM) en pacientes que no respondieron a terapias conservadoras, publicado por Reston y Turkelson. ${ }^{17}$ Estos autores, después de analizar más de 1.600 revistas y suplementos de una cuidadosa selección, llegaron a dos conclusiones: la primera parece ser que los pacientes que no responde al tratamiento médico obtienen cierto beneficio con el tratamiento quirúrgico y en los que tienen un desplazamiento del disco sin reducción, existe una evidencia relativa de que el tratamiento quirúrgico es más eficaz. La controversia a este artículo en la misma revista fue hecha por Laskin y Best, quienes cuestionan los métodos utilizados para realizar el MA, por lo que respecta a los posibles «sesgos», dada la mezcla inclusiva y exclusiva realizadas, así como la diversidad de parámetros tenidos en cuenta y la no consideración de aspectos clínicos, tiempo y características de los tratamientos conservadores así como y las diferencias quirúrgicas implícitas en las técnicas. Por todo ello y a pesar de su esfuerzo, dicen que alcanzan unas poco claras y decepcionantes conclusiones, que incluso podrían haberse expresado de otro modo más acorde con lo que debe trasmitírsele al cirujano, en el sentido de prudencia antes de un proceder quirúrgico en estos casos.

En lo que respecta a la metodología, diversos autores han demostrado que no hay diferencia entre las conclusiones de estudios observacionales correctamente planeados y ensayos clínicos randomizados también correctos sobre el mismo sujeto, Vandenbrouc$\mathrm{ke}^{18}$ Benson $^{19}$ y Concato. ${ }^{20}$ Cuando analizamos con espíritu crítico las publicaciones basadas en métodos empíricos, encontramos que frecuentemente las inclusiones de pacientes no han sido imparciales, que se han omitido datos, que a veces los métodos analíticos empleados en las mismas son incorrectos, que los resultados pueden ser tendenciosos por la manipulación de datos, ocultación de aspectos negativos, etc. Por ejemplo, un error grave es considerar la escala ordinal como una escala cuantitativa y realizar con la misma operaciones algebraicas, como hallar la media de una clasificación por grados diagnósticos (Virseda y Salinas). ${ }^{21}$ En resumen, «sesgos sistemáticos»que responden a lo que antes comentamos como falta de rigor científico, metodológico o incluso de honradez. Estos aspectos que, lamentablemente, aparecen en publicaciones con bastante frecuencia, son uno de los puntos clave en la defensa de la MBE. 
Cuando un fenómeno de cualquier tipo adquiere una relevancia pujante en la discusión científica, como es el caso de la metodología de la MBE en Ciencias de la Salud, hay que analizar las razones por las que esto ocurre. La filia hacia la MBE por parte de médicos, revistas científicas, industria farmacéutica, empresas de tecnología, gestores sanitarios, políticos o incluso jueces, tiene razones diversas y profundas, pero que en general podemos simplificar diciendo que existe una exigencia de parcelación, uniformización y transformación mensurable en todos los órdenes, de la complejidad que supone el amplio campo del ejercicio de la medicina.

Sin menospreciar el valor que merece y tiene la correcta conjunción de técnicas epidemiológicas y estadísticas en apoyo de la ciencia, que son tan útiles en probar la eficacia diagnostica o de tratamiento, hemos de dejar bien claro que también existe un empirismo perfectamente válido y que ha representado hasta el momento la spunta de lanza»de gran parte de los descubrimientos y avances científicos acontecidos en la medicina.

\section{Las publicaciones científico-médicas}

Los méritos científicos de la que podríamos denominar «carrera profesional»del médico, históricamente han tenido uno de sus pilares básicos en las publicaciones de su ámbito (revistas, monografías y libros), como expresión de su espíritu de trabajo, superación y capacidad de transmitir su creatividad en el campo profesional. Desde que un profesional inicia su formación se le induce a que anualmente publique una serie de trabajos científicos: casos clínicos, revisiones, tesis, estudios comparativos, etc., lo que conforma toda esa parafemalia de letra impresa a la que se liga toda la vida del médico.

Tenemos suficientes medios de difusión de la ciencia, apoyos financieros interesados y muchos profesionales ansiosos por utilizarlos, lo que sin duda ha repercutido en la calidad de las revistas científicas; mucho de lo que se publica es basura»científica, que solo sirve para el curriculum. Esto alcanza un significado muy especial en países como el nuestro, donde la accesibilidad médica al sistema sanitario público, casi monopolístico y la promoción profesional del medico, tienen una de sus bases de mayor speso»en baremos y puntuaciones de producción científica, sobre todo en función de criterios cuantitativos y no cualitativos.

La proliferación de publicaciones científicas, que se ha duplicado en los últimos 10 años, ha dado como resultado el que un clínico que siga únicamente las diez mejores publicaciones de Medicina Intema, tenga que leer una media mensual de 200 artículos y 10 editoriales para considerarse científicamente «al día». Por otra parte, los defensores de la MBE consideran que de los trabajos publicados hoy en día apenas el tres por ciento están elaborados con «rigon»metodológico. Esto ha llevado a que los médicos se sientan atraídos a una metodología que simplifica, uniformiza y, por ende, les ayuda en su ejercicio profesional, lo que ya comienza a ser demandado y puesto en práctica por algunas Facultades de Medicina (Bloch). ${ }^{22}$

\section{La alianza industria-académica}

Consecuentemente con la idea anterior, no es menos cierto también que la mayor parte de las sociedades científico-médicas y muchas iniciativas de grupos especializados, han luchado incesantemente por conseguir disponer de una plataforma editorial que aglutine las publicaciones especializadas y esté controlada por ellos. Si a todo ello le añadimos que la industria sanitaria (farmacéutica, tecnológica y de productos) representa una de las cifras macro-económicas más considerables a nivel de cualquier país y que el médico es sin lugar a dudas el mayor inductor de este consumo y al tiempo quien ha de validar casi todos los estudios, es fácil colegir que el apoyo de estos sectores se ha traducido en que no existe ninguna actividad, que disponga de tantas publicaciones como la Medicina. La industria farmacéutica precisa demostrar ante las agencias estatales reguladoras, la efectividad de sus productos antes de que sean introducidos en el mercado. Esta demostración requiere estudios clínicos para determinar falta de nocividad, tamaño de dosis y eficacia clínica, lo que conlleva una gran inversión financiera en las Organizaciones de Investigación Clínica -consultorías privadas de gestión- que diseñan y gestionan estos estudios y que, en general, obtienen beneficios muy superiores a los departamentos clínicos que realizan los ensayos clínicos. Como apunta Sackett, ${ }^{23}$ cada semana se publican más de 200 ensayos clínicos. Solo en EE.UU. hay más de 20 millones de personas que participan en ellos. En general los investigadores que participan en un ECR no tienen control sobre su diseño o sobre el análisis de los datos en los que se basan las conclusiones. La industria farmacéutica generalmente controla la metodología, el análisis de datos y las conclusiones finales, ignorando, a veces resultados desfavorables. Freiberg, ${ }^{24}$ Yaphe, ${ }^{25}$ y Weatherall, ${ }^{26}$ por separado, han demostrado una correlación directa entre la fuente de financiación de un estudio y la posibilidad que los resultados de un ECR sean favorables al producto fabricado por la entidad financiadora. Existe también otro tipo de sesgo en contra de los estudios que no son significativos, relativo a que las revistas científicas no son proclives a la publicación de estudios con resultados negativos. Al respecto de todo lo comentado, creemos que una referencia clave han sido las reflexiones publi- 
cadas por diversos editores de doce importantes revistas científicas intemacionales y reproducidos en alguna de nuestra revistas nacionales. ${ }^{27}$

\section{La filosofía utilitarista}

El Estado-Social, se ha ido perfilando en función de un modelo «utilitarista» de los recursos sanitarios, «o mejor para el mayor número», dejándose sentir su efecto paulatinamente en los últimos 25 años, tanto para la salud de la población en general, como en la cobertura de la asistencia sanitaria.

Los responsables de la política sanitaria, en general precisan de opciones numéricas que les sirvan para tomar determinadas decisiones sin la necesidad de depender del criterio individual o del equipo del profesional especializado y cuyo lenguaje y justificación pueden ser subjetivas, menos cuantificables, menos compresibles y por lo tanto limitadoras en sus opciones a la hora de dirigir el gasto sanitario. Una recopilación hecha por Booth, ${ }^{28}$ refleja diferentes iniciativas en gestión sanitaria con criterios de MBE, llevadas a cabo en países desarrollados, mientras que en otros subdesarrollados han servido para priorizar políticas de salud (Murray y Lopez), ${ }^{29}$ sobre todo a través del Meta-análisis (Mulrow); ${ }^{30}$ aunque Aveyard, ${ }^{31}$ estima que su empleo en temas de salud no es acertado, por cuanto considera más importante el resultado que el modo cualitativo en la toma de decisiones.

Estamos ante un sector considerado socialmente de primera necesidad, donde el gasto económico puede llegar a ser exponencial, sin que como contrapartida sus gestores tengan mecanismos adecuados tanto para su freno como para su control. Por su parte, los médicos, a través de un simple bolígrafo, tienen una capacidad asombrosa de inducir gasto; las nuevas tecnologías, publicitadas adecuadamente por las empresas, crecen y son demandadas tanto por los médicos como por los pacientes. Los laboratorios farmacéuticos en su dinámica comercial influyen tanto sobre el consumidor directo como sobre el médico como prescriptor, dando como resultado que sea el gasto hasta el momento de mayor dificultad para su control.

Todo ello nos lleva a gestores y profesionales a un enfrentamiento permanente; los profesionales, también contribuyentes, entienden los modelos de gestión sanitaria y los cumplen, pero no abdican del derecho la crítica a de los mismos cuando estiman que comprometen negativamente su ejercicio profesional en general o sobre pacientes concretos. A este entramado socio-sanitario tenemos también que añadirle un elevado nivel de judicialización sanitaria, cada día más elevado, y que en países como EE.UU., esta fuera de control, lo que lleva a los tribunales de justicia a simplificar y encasillar los problemas médicos para la mejor compresión de las demandas. Los médicos por su parte, en su necesidad de cubrirse en lo posible las espaldas, inducen un gasto sanitario añadido e innecesario para una buena práctica profesional, pero a veces exigido a instancias pseudocientíficas, la «Medicina Defensiva».

Interesantes estimamos las reflexiones de Rosenfeld, ${ }^{32}$ en la revista ORL y Cirugía de Cabeza y Cuello de Enero de este año. Considera que la medicina está basada siempre en la «incertidumbre». Asimismo, piensa que es preciso aprovechar las herramientas cuantificadoras en lo que puedan sernos útiles, para alcanzar nuevos niveles de probabilidad que no suponen que sean verdades absolutas. Si bien en todo proceso evolutivo científico la metodología de la MBE tiene un papel positivo y posiblemente más importante en el futuro de concretos campos de la medicina, no se puede consentir que se sojuzgue el método empírico-inductivo en otros aspectos de la investigación médica. Osler, ${ }^{33}$ en su escepticismo, manifestaba ya la idea de que la buena práctica clínica es una mezcla del arte de la incertidumbre, con la ciencia de la probabilidad. Martín-Granizo, ${ }^{34}$ aboga por el equilibrio entre la evidencia y la experiencia; para Naylor, ${ }^{35}$ la medicina es más compleja que la aplicación de reglas científicas y la MBE es poco útil cuando se enfrenta a lo que llama «onas grises» de la práctica, con evidencia de riesgos y opciones clínicas incompletas o contradictorias. La buena evidencia va más allá del empleo de MA y de los ECR, del mismo modo que no se libera del juicio personal (Green y cols.). 36

En un divertido y polémico artículo, Graham-Smith, ${ }^{37}$ establece un diálogo entre un entusiasta de la MBE, al que llama «Entusiasticus Metanalyticus»y Sócrates; el primero o «cuantificador»intenta ganarse al escéptico filósofo con los encantos de la aplicación de la ciencia globalizada, pero cuando Sócrates no llega más que a entender que ello pueda ser un conflicto entre el «arte»y la ciencia de la Medicina, su polemista confiesa su adicción por la metodología y reconoce que no tiene tiempo para ver a sus pacientes.

\section{Conclusiones}

La cruda y dura realidad es que hasta el momento actual el ejercicio de la medicina empírica evolucionada tiene el bagaje de haber conseguido los índices de morbi-mortalidad y los niveles de calidad de vida que disfrutamos, mientras que corresponde a la MBE, conseguir demostrar que su aplicación repercute en la mejora de los índices que realmente cuentan, una mayor y más larga vida; tal beneficio de la MBE queda por demostrar, como reconocen sus propios defensores (Salinas y Virseda), ${ }^{38}$ y (Strauss). ${ }^{39}$ 


\section{Bibliografía}

1. Goodman S. Towards Evidence-based Medical Statistic. 1: the p-value fallacy. Ann Int Med 1999;13:995.

2. Goodman S. Towards Evidence-based Medicine Statistic. 2: the Bayes factor. Ann Int Med 1999;130:1005.

3. Cochrane AL Effectiveness and efficiency. Random reflexions an health services. Nuffield Provincial Hospitals Trust. London 1972.

4. Sackett DT,Gent M. Controversy in counting and attributing events in clinical trials. N Engl J Med 1979; 301:1410.

5. Sackett DL, Rosenberg WMC, Muir Gray JM, Haynes RB, Richardson WS. Evidence based medicine: what it is and what it isn't. B Med J 1996;312:71.

6. Harrington JT. Primary things firts. Acad Med 1997;72:938.

7. Jadad A. Randomized Clinical Trials. BMJ- Boocks. London, 1998.

8. Labreze L. Evidence based medicine: a tool for quality. Hospital 2002;4:10.

9. Estrada MD y Oliva G. Informatiu AATRM 2003;29:20.

10. Eysenck HJ. Meta-analysis and its problems. BMJ 1994; 309:789

11. Schlemper RJ, y cols. Differences in diagnostic criteria for gastric carcinoma between japanese and western pathologists. Lancet 1997;349:1725.

12. The Executive Committee for the Asymptomatic Carotid Atherosclerosis study: Endarterectomy for asymptomatic carotid stenosis. JAMA 1995;273:1421.

13. North American Symptomatic Carotid Endarterectomy Trial (NASCET) Steering Committee. North American Symptomatic Carotid Endarterectomy trial: Methods, patient characteristics and prognosis. Stroke 1991;22:711.

14. Imparato AM, Riles TS, Mintzer R, Bauman FG. The importance of hemorrhage in the relationship between gross morphologic characteristics and cerebral symtoms in 376 carotid artery plaques. Ann Surg 1982;2:195.

15. Dixon S, Pais SO, Raviola C y cols. Natural history of non-stenotic asymptomatic ulcerative lesions of the carotid artery. Arch Surg 1982;117:1493.

16. Geroulakos G, Domjan J, Nicolaides A y cols. Ultrasonic carotid artery plaque structure and the risk of cerebral infarction on computed tomography. J Vasc Surg 1994; 20:263.

17. Reston JT, Turkelson CM. Meta-Analysis of surgical treatments for temporomandibular articular disorders. J Oral Maxillofac Surg 2003;61:3.

18. Vandenbroucke JP. In defense of case reports and case series. Ann Intern Med 2001;134:330.

19. Benson K, Hartz AJ. A comparison of observational studies and randomized controlled trials. N Engl J Med 2000;342:1878.
20. Concato y cols. Randomized, controlled trials, observational studies, and the hierarchy of research designs. N Engl J Med 2000;342:1887.

21. Virseda M, Salinas J. Teoría de la Investigación: el método científico en Ciencias de la Salud. Urol Integr Invest 2001;6:247.

22. Bloch RM, Swanson MS, Hannis MD. An extended evidence-based medicine curriculum for medical students. Acad Med 1997;72:431.

23. Sackett DL, Hoey J. Why randomized controlled trials fail but needn't: a new series is launched. CMA J 2000;162: 1301.

24. Freidberg M, Saffran B, Stinson TJ, nelson TJ and Bennett CL. Evaluation of conflict of interest in economic analyses of new drugs used in oncology. JAMA 1999; 282;1453.

25. Yaphe J, Edman R, Knishkowy B, Herman J. The association between funding by commercial interests and study outcome in randomized controlled drug trials. Fam Practice 2001;18:565.

26. Weatherall D. Academia and industry: increasing uneasy bedfellows. Lancet 2000;355:1574.

27. Editorial. Rev Esp Cardiol, 2001;54:1247.

28. Booth A. How evidence based is your practice?. Hospital 2002;4:14.

29. Murray CJL, Lopez AD, Evidence-based health policy, lesson from the global burden of disease study. Science 1996,274:740.

30. Mulrow CD. Rationale for systematic reviews. BMJ 1994; 309:597.

31. Aveyard P. Evidence-based medicine and public health. J Eval Clin Pract 1997;3:139.

32. Rosenfeld RM. Comentary: uncertainty-based medicine. Otolaryngol Head Neck Surg 2003;128:5.

33. Osler W. On the educational value of the medical society. En: Aequanimitas With Other Addresses. P Blakiston's Son and Co., Philadelphia, 1904; pg. 348.

34. Martín-Granizo R. MBE. Medicina Basada en dila Evidencia o la Experiencia? Rev Esp Cirug Oral y Maxilofac 2001; 23:269.

35. Naylor CD, Lopez AD. Grey zones of clinical practice: some limits to evidence-based medicine. Lancet 1995; 345:840.

36. Green J, Torres TT, Alonso P. Quality research and evidence based medicine. BMJ 1998;316:1230.

37. Grahamme-Smith D. Evidence based medicine:socratic dissent. BMJ 1995;310:1126.

38. Salinas J, Virseda M. Arch Esp Urol 1999;52(8):817

39. Straus SE. Evidence-based Medicine: a commentary on common criticisms. CMAJ, 2000;163:837. 